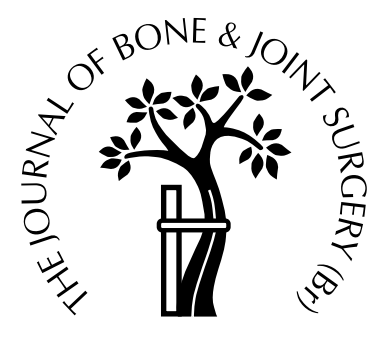

Aspects of current management

\title{
THE EFFECTS OF BOTULINUM TOXIN (BTX-A) ON SPASTICITY OF THE LOWER LIMB AND ON GAIT IN CEREBRAL PALSY
}

\author{
R. A. Preiss, D. N. Condie, D. I. Rowley, H. K. Graham \\ From Ninewells Hospital, Dundee, Scotland and Royal Children's Hospital, Melbourne, \\ Australia
}

Spasticity in cerebral palsy stems from a net disinhibition of spinal reflexes because of a primary non-progressive cerebral lesion. The resulting hyperactive reflexes and increased muscle tone create a dynamic, shortening deformity which is exacerbated by longitudinal skeletal growth in the growing child. This makes the management of dynamic deformities such as an equinus gait by physiotherapy and orthoses, particularly difficult during growth spurts.

The objectives of management in spasticity are to optimise functional movement and prevent fixed deformity such as joint contracture and bony torsion. Botulinum toxin (BTX-A) has been used since the early nineties in the management of focal spasticity in cerebral palsy. ${ }^{1}$ It is a clostridial neurotoxin which achieves a reduction in tone and spasticity in a targeted muscle by temporary disruption of acetylcholine exocytosis at the motor endplate. The resulting reduction of overactivity seen in an injected muscle facilitates the function of its antagonists, allowing a change in muscle balance which may make joint movement easier.

Of the seven serotypes (A-G) of botulinum toxin, serotypes A, B and F have been used in clinical practice. BTX-A has the longest duration of action, the greatest potency and is the most relevant serotype. There are significant differences between Botox (Ipsen Ltd, Slough, Berkshire, UK) and Dysport (Allergan Inc, Irvine, California), the two commercially available preparations of type-A botulinum toxin, in terms of potency and therefore dosage, total protein load and antigenicity. ${ }^{2}$

Recovery of muscle weakness after injection of BTX-A occurs in two stages. First, functional sprouts appear at the motor nerve terminal which re-establish nerve conduction.

R. A. Preiss, FRCS, Research Fellow in Orthopaedic Surgery D. N. Condie, Honorary Senior Lecturer

D. I. Rowley, MD, Professor and Head of Academic Department

c/o TORT Centre, Ninewells Hospital, Dundee DD1 9SY, UK.

H. K. Graham, MD, Professor and Director of Orthopaedic Surgery Royal Children's Hospital, Flemington Road, Parkville, Melbourne, 3052 Victoria, Australia.

Correspondence should be sent to Mr R. A. Preiss.

C)2003 British Editorial Society of Bone and Joint Surgery

doi:10.1302/0301-620X.85B7.13967 \$2.00

J Bone Joint Surg [Br] 2003;85-B:943-8.
This is followed by resumption of exocytosis at the original endplate and an eventual return of the motor endplate to its preinjection state, ${ }^{3}$ with a clinical return of spasticity at from 12 to 16 weeks in most patients.

\section{Indication}

The indication for the use of BTX-A injection is a dynamic spasticity, interfering with function, in the absence of fixed deformity. ${ }^{4}$ Passive correction of the deformity should give a range of movement of a joint which will allow relatively normal gait. A failure to respond to a tone-reducing cast or an inability to gain orthotic control over the deformity strengthens this indication further. This usually follows the growth spurt at around five years of age when skeletal growth outstrips that of the resisting soft tissues. The improvement of a dynamic deviation of gait to injection of BTX-A depends on the proportion of spastic deformity to fixed deformity. In general, younger children have more spasticity and less fixed contracture than older children and therefore respond more to BTX-A. ${ }^{3}$ Some authors have recommended injections in children of four years of age and younger in the hope of preventing or delaying the onset of fixed deformity. ${ }^{5}$

Clinicians should be aware of the approved or licensed indications in their country and medical system. Families should be informed if injection with BTX-A is outside the terms of current approval. All such 'off-label' use should be part of ethically approved clinical trials.

\section{Protocol and dosage of injection}

There are two distinct approaches to injection, depending on local arrangements and the preferences of the family and the clinician. Orthopaedic surgeons with access to anaesthetic expertise may prefer light general anaesthesia and muscle stimulation to identify target muscles. This approach is necessary for smaller, deeply placed muscles such as tibialis posterior. Other clinicians have reported a successful outcome after injections to large, superficial muscles, such as the gastrocnemius, targeted by manual palpation and using anaesthetic cream and/or oral sedation. ${ }^{4}$

Calculation of the dose takes into account the mass of the child, the number of muscles targeted, and the commercial 
Table I. A management algorithm for hemiplegia according to Winters et al ${ }^{11}$

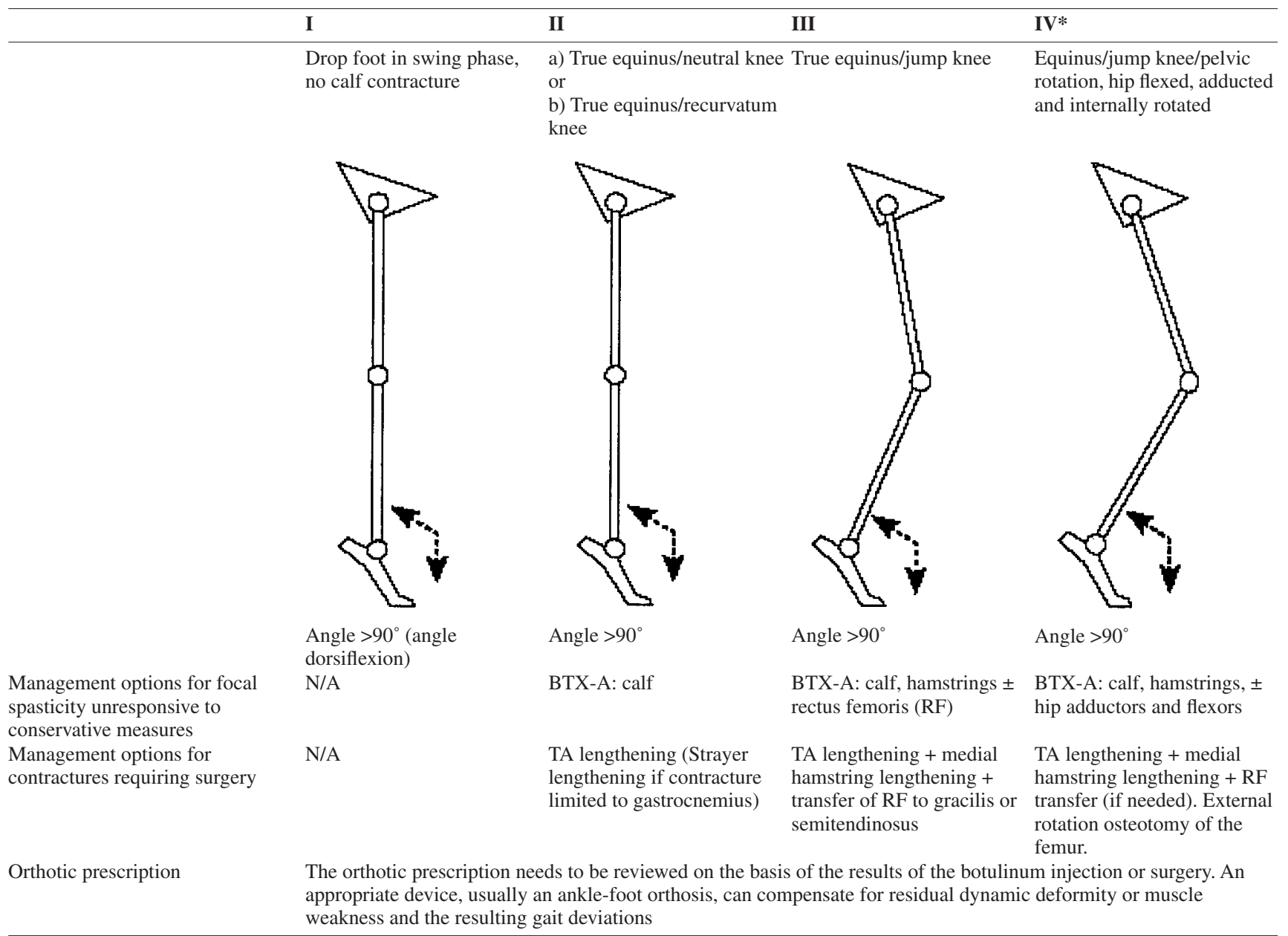

*RF, rectus femoris; TA, tendo achilles

preparation chosen. In multilevel injections, the dosage of BTX-A per muscle or the number of sites targeted may be restricted by a total permissible dose, as determined by the body mass. The two BTX-A preparations, Botox ${ }^{\circledR}$ and Dysport ${ }^{\circledR}$, are not equivalent in terms of dose with an approximate ratio of 1:3-4. Typically, a muscle in the lower limb receives 3 to $6 \mathrm{U} / \mathrm{kg}$ of Botox or the Dysport equivalent. For injections into gastrocsoleus, Baker et $\mathrm{al}^{6}$ have recommended $20 \mathrm{U} / \mathrm{kg}$ of Dysport divided between the two calves.

If early contracture is thought to be present, serial casting may be considered immediately after injection or after two weeks. Otherwise, children continue to use their orthoses. All recipients of BTX-A are entered into an intensive programme of physiotherapy so that the functional benefits may be extended beyond the clinical effect of the injection.

\section{Side-effects}

BTX-A can diffuse across fascial planes ${ }^{7}$ and intra-axonally, ${ }^{8}$ making distant side-effects a concern. Nevertheless, the standard doses of BTX-A have a safe clinical profile. ${ }^{9}$
As with the clinical effect, the side-effects of BTX-A are temporary and dose- and site-related.

The side-effects may be gait-related or more general. Gait-related problems may include non-response, overcorrection, rebound effects, deterioration in gait, local weakness and falls. Side-effects which are not gait-related occur in between $6 \%$ and $12 \%$ of treatments. The most serious is aspiration pneumonia in children with spastic quadriplegia and pre-existing pseudobulbar palsy. The systemic spread of very small amounts of BTX-A may be enough to impair pharyngeal function further in these children. A history of pseudobulbar palsy, gastro-oesophageal reflux and frequent chest infections are contraindications to the use of BTX-A. Other side-effects include local pain, global weakness, muscle atrophy and, rarely, urinary and faecal incontinence. The sequential administration of BTX-A can provoke an immune response with the production of antibodies. ${ }^{10}$ However, there is currently no evidence to suggest that subsequent non-response may result. Clinicians administering BTX-A should be aware that aminoglycosides and nonpolarising muscle relaxants cause potentiation of the neuromuscular blockade. 
Table II. A management algorithm for diplegia according to Rodda et al ${ }^{12}$

\begin{tabular}{|c|c|c|c|c|}
\hline & I & II & III & IV* \\
\hline & True equinus & $\begin{array}{l}\text { Jump gait (with or without } \\
\text { stiff knee) }\end{array}$ & $\begin{array}{l}\text { Apparent equinus (with or } \\
\text { without stiff knee) }\end{array}$ & $\begin{array}{l}\text { Crouch gait (with or without } \\
\text { stiff knee) }\end{array}$ \\
\hline & Angle $>90^{\circ}$ (angle & Angle $>90^{\circ}$ & Angle $=90^{\circ}$ & Angle $<90^{\circ}$ \\
\hline $\begin{array}{l}\text { Management options for focal } \\
\text { spasticity unresponsive to } \\
\text { conservative measures }\end{array}$ & BTX-A: calf & BTX-A: calf, hamstrings & $\begin{array}{l}\text { BTX-A: hamstrings and } \\
\text { iliopsoas in younger patients }\end{array}$ & $\begin{array}{l}\text { BTX-A: hamstrings and } \\
\text { ilipsoas in younger patients }\end{array}$ \\
\hline $\begin{array}{l}\text { Management options for } \\
\text { contractures and skeletal } \\
\text { deformity requiring surgery }\end{array}$ & TA lengthening & $\begin{array}{l}\text { SEMLS to address fixed } \\
\text { contractures and lever arm } \\
\text { disease }\end{array}$ & $\begin{array}{l}\text { SEMLS to address fixed } \\
\text { contractures and lever arm } \\
\text { disease }\end{array}$ & $\begin{array}{l}\text { SEMLS to address fixed } \\
\text { contractures and lever arm } \\
\text { disease }\end{array}$ \\
\hline Orthotic prescription & $\begin{array}{l}\text { The orthotic prescr } \\
\text { appropriate orthosi } \\
\text { deviations. }\end{array}$ & $\begin{array}{l}\text { ds to be reviewed on the bas } \\
\text { pensate for residual dynamic }\end{array}$ & $\begin{array}{l}\text { of the results of the botulinum } \\
\text { eformity or muscle weakness }\end{array}$ & $\begin{array}{l}\text { injection or surgery. An } \\
\text { and the resulting gait }\end{array}$ \\
\hline
\end{tabular}

*SEMLS, single event multi-level surgery

\section{Applications of BTX-A in the lower limb in cerebral palsy}

Although patterns of gait in spastic motor disorders are variable, four basic patterns in the sagittal plane have been described in spastic hemiplegia by Winters, Gage and Hicks $^{11}$ (Table I). More recently, Rodda et $\mathrm{al}^{12}$ have described four sagittal patterns in spastic diplegia (Table II). Given that the major muscle groups in the lower limbs have their principal action in the sagittal plane, such deviations are most relevant to the management of spasticity and contracture. Both classifications have been instrumental in allowing the development of a problem-orientated approach by providing a template for the management of spasticity. ${ }^{12}$ However, they allow for neither deformity in the coronal plane secondary to discrepancy in limb length and subluxation of the hip, nor deformity in the transverse plane which may result from pelvic rotation and bony torsion. Patients with deformities in these planes warrant investigation at experienced centres which are able to perform three-dimensional gait analysis.

Gastrocnemius-soleus complex. The most common abnormality of gait in cerebral palsy is an equinus gait. The kinematics of the ankle in the normal stance phase are described in terms of three rocker phases. During the first rocker phase, the ankle plantar flexes, and the foot is lowered to the ground under the control of the eccentrically acting tibialis anterior. In the second, progressive dorsiflexion of the ankle allows the tibia and the centre of gravity to progress over the fixed foot. This is modulated by the eccentrically acting triceps surae. During the third, the foot rotates over the metatarsal heads and the concentric contraction of the triceps surae and flexor hallucis provides push-off.

In equinus gait the first rocker phase is usually absent because of initial contact by a toe or flat foot. In the second phase, loading of the body-weight on to the ankle and foot causes lengthening of the gastrocsoleus which is terminated abruptly by a reflex contraction or contracture of the spastic gastrosoleus. Ankle kinematics show a typical 'doublebump' pattern because the ankle returns inappropriately to the equinus range. This abnormal gastrocsoleus contraction in response to lengthening compromises the ability to generate push-off during the third rocker phase. During the swing phase, the equinus position of the foot may impair clearance and result in tripping.

Injection of BTX-A for true equinus may be indicated in both spastic hemiplegia and in spastic diplegia, in which the involvement is peripheral and relatively isolated to the gastrocsoleus (Winters group II; Rodda and Graham group I), 

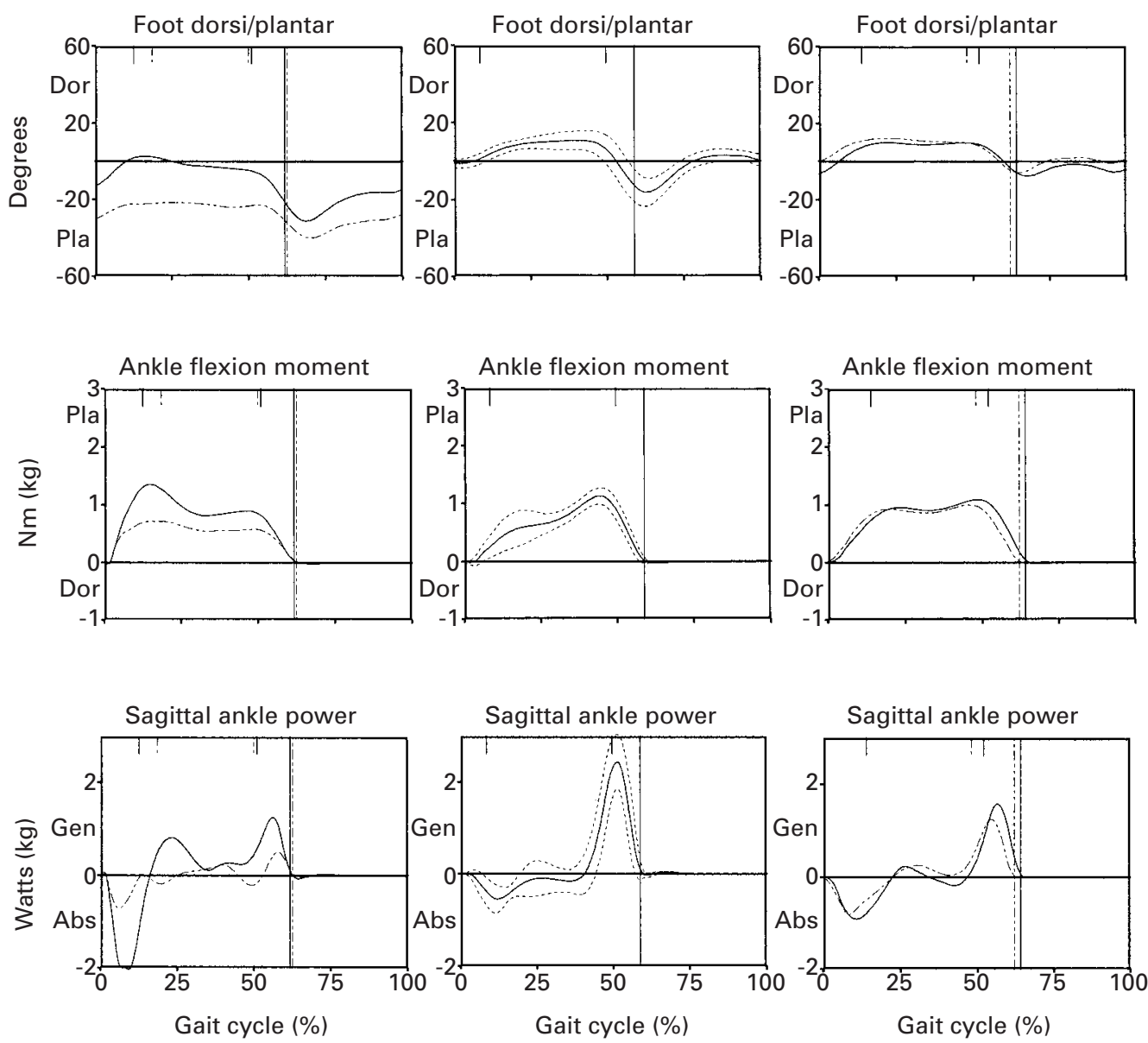

Pre-BTX-A injection

Normal range

Post-BTX-A injection

Fig. 1

\begin{abstract}
A six-year-old boy with asymmetric diplegia presented with toe-walking and brace intolerance. Clinical examination suggested that this was mainly the result of gastrocnemius spasticity. His ankle kinematics traces (left-hand column) confirmed equinus gait worse on the right (solid line) than the left (dotted line). Ankle kinetics confirmed a double-bump pattern and the power curves were triphasic, with poor push-off in terminal stance. He was managed by injection of Botox, 4 units $/ \mathrm{kg}$ to each calf muscle and had a cast applied to the right foot and ankle for a residual $10^{\circ}$ fixed equinus deformity, for one week. He had a follow-up gait analysis two months after injection (right-hand column). He was no longer toe-walking and could wear his AFOs comfortably. Both ankle kinematics traces have moved from equinus into a more normal range. Ankle kinetics are also improved with a paradoxical increase in power generation at push-off.
\end{abstract}

and in younger diplegics in whom the equinus foot is usually transient.

BTX-A allows increased muscle length, as shown by the model of Eames et al, ${ }^{13}$ and significantly increased dorsiflexion of the ankle. This gives improved ground clearance for advancement of the limb, and, subsequently, better initial contact of the foot. The reflex contraction of the triceps surae is ameliorated, which is reflected by a reduction in the double-bump phenomenon during the second rocker (phase) (Fig. 1). Paradoxically, given the weakening effect of BTX$A$, power generation at push-off may increase ${ }^{14}$ with improved length and velocity of the stride. This has been termed the 'biomechanical transformation' of gastrocsoleus.

BTX-A gastrocsoleus injections are also used to treat the equinus component of the more complex gait pattern disor- ders in spastic hemiplegia and spastic diplegia, which are characterised by multi-level involvement (Winters group II, III and IV; Rodda and Graham group II). These complex gait disorders may benefit from a multilevel approach of BTX injections as developed by Molenaers et $\mathrm{al}^{15}$ in Leuven, Belgium, in which a combination of selected muscles is targeted at one sitting. They emphasise the use of instrumented gait analysis before intervention in order to define the objectives of treatment. Aggressive casting, night splints and the use of appropriate orthoses follow multilevel injections of BTX-A to specific target muscles. They then perform a further gait analysis to determine the outcome of their management. This approach requires detailed knowledge of spastic gait disorders, abnormalities of gait and a rigorous approach to both the injection programme and 
after-care. Very large total doses of BTX-A are used, which seem to be safe when specific guidelines are followed. This approach is not for the inexperienced. No regulatory authorities have approved BTX-A to be used in this manner and all such investigations should be in the hands of experienced users in approved clinical trials.

A good clinical response to injection of gastrocsoleus for equinus gait is shown by a resolution or decrease in toewalking. This has been quantified by the significant improvements reported in the gross motor function measure, which examines the ability to perform basic movements and the physicians rating scale, a video-derived gait score. Subsequent meta-analysis has confirmed that the use of BTX-A for an equinus gait disorder is of moderate additional benefit when compared with serial casting or placebo. $^{16}$

Tibialis posterior. Botulinum toxin has a diagnostic and therapeutic role in the dynamic equinovarus deformity of the hindfoot. This can be due to unopposed activity of the tibialis posterior, tibialis anterior or triceps surae. Should injection of the triceps surae fail to correct the equinovarus, injection of tibialis posterior may be performed. A temporary collapse into hindfoot valgus has been reported after successful injection. ${ }^{17}$ It is suggested that this confirms involvement of the tibialis posterior.

Hamstrings. Spasticity of the hamstrings is a primary feature in diplegia (Rodda and Graham groups II, III and IV) and is also seen in more severe manifestations of hemiplegia (Winters groups III and IV). ${ }^{12}$ Hamstring spasticity resists normal knee extension during late stance, and in association with spasticity of the iliopsoas, may result in crouch gait.

Thompson et $\mathrm{al}^{18}$ and Corry et $\mathrm{al}^{19}$ have reported the results of injections of BTX-A into the hamstrings of children with a crouch gait. An increased range of knee movement and an increase in extension of the knee at initial contact and mid-stance were observed. Modest improvements in function of the hip flexors and in the range of hip movement were also seen. When the hamstrings were not demonstrably shortened, there was a tendency towards increased anterior pelvic tilt. Injection can have a useful role as a predictor of the results of lengthening of the hamstring tendons.

Adductors. Spasticity of the adductors forms part of the clinical picture in quadriplegia as well as in the more severe manifestations of hemiplegia (Winters group IV) and diplegia (Rodda and Graham group III and IV). So far, there is little evidence to show that injection for adductor spasticity results in improvement of gait, although such injection is often included in a multilevel approach in these conditions. However, improvements in positioning, orthotic tolerance and perineal access for hygiene have been noted in patients who are unable to walk. ${ }^{20}$ Perception of improvement by their carers may be as good an assessment as any defined outcome measure in this group of patients.

Rectus femoris. Concomitant spasticity of rectus femoris and the hamstrings results in a flexed stiff knee gait which may co-exist with other patterns of abnormality. In diplegia this includes Rodda and Graham groups II, III and IV and in hemiplegia, Winters groups III and IV. Normally, rectus femoris works concentrically to flex the hip during push-off and eccentrically at the knee to control the rate of flexion during the swing phase. Although injections into rectus femoris are not performed commonly, improvement in the velocity of knee flexion and in maximum knee flexion has been observed. ${ }^{21}$ Good clinical judgement is required in the marginal walker, since spasticity in the quadriceps may be essential in some children in order to maintain knee extension during stance, and hence an upright posture.

Iliopsoas. Dynamic deformity of hip flexion during gait is seen in most children with diplegia (Rodda and Graham groups II, III and IV) and in those with more severe hemiplegia (Winters group IV). Spasticity of the iliopsoas muscle inhibits the ability of the hip to assume an extended position in stance. Gait analysis before injection shows an abnormal increase in the velocity of the hip flexion in the pre-swing and initial swing phases, and decreased extension in the stance phase. These parameters are reported to improve after injection of BTX-A. ${ }^{15}$ The injection is performed with the needle entering adjacent to the anterior superior iliac spine and is technically demanding. General anaesthesia and muscle stimulation are advisable and ultrasound guidance has been used successfully. ${ }^{22}$

Hip subluxation or dislocation. Theoretically, it may be possible to modify the natural history of subluxation or dislocation of the hip by reducing spasticity in the iliopsoas and adductors over an extended period. Boyd et $\mathrm{al}^{23}$ described a trial in the 'at-risk' hip which evaluated the combination of injections of BTX-A into the iliopsoas and adductors and a hip abduction brace. Fewer patients required adductor tenotomy at a follow-up of one year. Increased tolerance of the brace was also noted. ${ }^{23}$ Botulinum toxin administered to the adductors before tenotomy has been shown to provide useful analgesia. ${ }^{24}$

\section{Conclusion}

The two main goals of BTX-A therapy are to correct abnormalities of gait and to delay or reduce the requirement for orthopaedic surgery.

The unique relationship between spasticity and strength in any spastic limb determines whether its function will benefit from BTX-A therapy. Two considerations are pertinent. First, the neuromuscular junctions which are suppressed to manage spasticity are the same as those which provide meaningful movement. Secondly, functional movement is also hindered by other elements of the upper motorneurone syndrome such as weakness and sensory impairment, which cannot be treated with BTX-A. Therefore, suitable patients need to have sufficient strength to withstand the inherent weakening effects of this treatment.

Although short- to medium-term evidence suggests that injection of the gastrocsoleus with BTX-A results in 
improvement in equinus, the effect of injection in the more proximal muscles is not yet clear.

Early referral of potential candidates for BTX-A therapy is essential since the greatest opportunity to delay or to prevent dynamic deformity from progressing to 'myostatic contracture' is before the age of four years. The ability of BTX-A therapy to delay surgery has been described. ${ }^{25}$ This may allow multilevel operations to be carried out on a single occasion when the gait is mature and recurrence of deformity less likely. Some patients may not need surgery. The prevention of contractures demonstrated in animal models, ${ }^{26}$ has suggested that such disease-modifying benefits are indeed possible.

\section{References}

1. Koman LA, Mooney JF 3rd, Smith B, Goodman A, Mulvaney T Management of cerebral palsy with botulinum-a toxin: preliminary investigation. J Pediatr Orthop 1993;13:489-95.

2. Aoki KR, Guyer B. Botulinum toxin type A and other botulinum toxin serotypes: a comparative review of biochemical and pharmacological actions. Eur J Neurol 2001;8(Suppl 5):21-9.

3. de Paiva A, Muenier FA, Molgo J, Aoki KR, Dolly JO. Functional repair of motor endplates after botulinum neurotixin type A poisoning: biphasic switch of synaptic activity between nerve sprouts and their parent terminals. Proc Natl Acad Sci USA 1999;96:3220-5.

4. Cosgrove AP, Corry IS, Graham KH. Botulinum toxin in the management of the lower limb in cerebral palsy. Dev Med Child Neurol 1994;36:386-96

5. Graham HK, Aoki KR, Autti-Ramo I, et al. Recommendations for the use of botulinum toxin type A in the management of cerebral palsy. Gait Posture 2000;11:67-79.

6. Baker R, Jasinski M, Maciag-Tymecka I, et al. Botulinum toxin treatment of spasticity in diplegic cerebral palsy: a randomized, double-blind placebo-controlled, dose-ranging study. Dev Med Child Neurol 2002;44:666-75.

7. Shaari CM, George E, Wu BL, Biller HF, Sanders I. Quantifying the spread of botulinum toxin through muscle fascia. Laryngoscope 1991;101:960-4.

8. Wiegand H, Erdmann G, Wellhoner HH. 125I-labelled botulinum A neurotoxin: pharmacokinetics in cats' intramuscular injection. Naunym Schmiedebergs Arch Pharmacol 1976;292:161-5.

9. Bakheit AM, Severa S, Cosgrove A, et al. Safety profile and efficacy of botulinum toxin A (Dysport) in children with muscle spasticity. Dev Med Child Neurol 2001;43:234-8.
10. Hermann J, Mall V, Bigalke H, et al. Secondary non-response due to development of neutralising antibodies to botulinum toxin A during treatment of children with cerebral palsy. Neuropediatrics 2000;31: 333-4.

11. Winters TF Jr, Gage JR, Hicks R. Gait patterns in spastic hemiplegia in children and young adults. J Bone Joint Surg [Am] 1987;69-A: 437-41.

12. Rodda JM, Graham HK, Galea MP, et al. Sagittal gait patterns in spastic diplegia. J Bone Joint Surg [Br] 2003; in press.

13. Eames NW, Baker R, Hill N, et al. The effect of botulinum toxin A on gastrocnemius length: magnitude and duration of response. Dev Med Child Neurol 1999;41:226-32.

14. Boyd RN, Pliatsios V, Starr R, Wolfe R, Graham HK. Biomechanical transformation of the gastroc-soleus muscle with botulinum toxin $\mathrm{A}$ in children with cerebral palsy. Dev Med Child Neurol 2000;42:32-41.

15. Molenaers G, Eyssen M, Desloovere K, Jonkers I, De Cock P. A multilevel approach to botulinum toxin type A treatment of the (ilio)psoas in spasticity in cerebral palsy. Eur J Neurol 1999;6(Supp14):59-62.

16. Boyd RN, Hays RM. Current evidence for the use of botulinum toxin type A in the management of children with cerebral palsy: a systematic review. Eur J Neurol 2001;8(Suppl 5):1-20.

17. Eyssen M, Molenaers G, Desloovere K, De Cock P. Equinovarus deformity of the foot in cerebral palsy: botulinum toxin A infiltration including M. Tibialis Posterior. Gait Posture 1999;10:74.

18. Thompson NS, Baker RJ, Cosgrove AP, Corry IS, Graham HK. Musculoskeletal modelling in determining the effect of botulinum toxin on the hamstrings of patients with crouch gait. Dev Med Child Neurol 1998;40:622-5

19. Corry Is, Cosgrove AP, Duffy CM, Taylor TC, Graham HK. Botulinum toxin A in hamstring spasticity. Gait Posture 1999;10:206-10.

20. Mall V, Heinen F, Kirschner J, et al. Evaluation of botulinum toxin A therapy in children with adductor spasm by gross motor function measure. J Child Neurol 2000;15:214-7.

21. Brooksbank AJ, Gibbs S, MacLean JGB. The alteration of knee kin ematics following injection of botulinum toxin into the rectus femoris of patients with spastic cerebral palsy. British Orthopaedic Research Society Meeting 2001.

22. Willenborg MJ, Shilt JS, Smith BP, et al. Technique for iliopsoas ultrasound-guided active electromyography-directed botulinum A toxin injection in cerebral palsy. J Pediatr Orthop 2002;22:165-8.

23. Boyd RN, Dobson F, Parrott J, et al. The effect of botulinum toxin type A and a variable hip abduction orthosis on gross motor function: a randomized controlled trial. Eur J Neurol 2001;8(Suppl 5):109-19.

24. Barwood S, Baillieu C, Boyd R, et al. Analgesic effects of botulinum toxin A: a randomized, placebo-controlled clinical trial. Dev Med Child Neurol 2000;42:116-21.

25. Koman LA, Smith BP, Tingey CT, et al. The effect of botulinum toxin type A injections on the natural history of equinus foot deformity in paediatric cerebral palsy patients. Eur J Neurol 1999;6(Suppl 4):19-22.

26. Cosgrove AP, Graham HK. Botulinum toxin A prevents the development of contractures in the hereditary spastic mouse. Dev Med Child Neurol 1994;36:379-85. 